\title{
Controlling the Polarization State of Light with a Dispersion-Free Metastructure
}

\author{
Shang-Chi Jiang, ${ }^{1}$ Xiang Xiong, ${ }_{1}^{1}$ Yuan-Sheng Hu, ${ }^{1}$ Yu-Hui Hu, ${ }^{1}$ Guo-Bin Ma, ${ }^{1}$ \\ Ru-Wen Peng, ${ }^{1}$ Cheng Sun, ${ }^{2}$ and Mu Wang ${ }^{1 *}$ \\ ${ }^{1}$ National Laboratory of Solid State Microstructures and Department of Physics, \\ Nanjing University, Nanjing 210093, China \\ ${ }^{2}$ Department of Mechanical Engineering, Northwestern University, Evanston, Illinois 60208-3111, USA
}

(Received 31 October 2013; published 15 May 2014)

\begin{abstract}
By combining the advantages of both a metallic metamaterial and a dielectric interlayer, we demonstrate the general mechanism to construct the dispersion-free metastructure, in which the intrinsic dispersion of the metallic structures is perfectly cancelled out by the thickness-dependent dispersion of the dielectric spacing layer. As examples to apply this concept, a broadband quarter-wave plate and a half-wave plate are demonstrated. By selecting the structural parameters, the polarization state of light can be freely tuned across a broad frequency range, and all of the polarization states on the Poincare sphere can be realized dispersion free.
\end{abstract}

DOI: 10.1103/PhysRevX.4.021026

Subject Areas: Metamaterials

Metamaterials are the artificial structures assembled with subwavelength building blocks featuring some physical properties that do not exist in the natural world $[1,2]$. Over the past decade, developments in this area have been characterized by the realization of numerous novel optical properties, such as negative refractive indices [3-5], superlenses [6-8], etc. A two-dimensional metamaterial offers the possibility of controlling light with miniaturized devices, which are essential, especially for integrated photonics $[9,10]$. Furthermore, by engineering the phase discontinuity on an interface, one can fully steer light and accomplish unparalleled control of anomalous reflection and refraction [11-15] and realize different optical devices, such as optical vortex plates [11,16] and wave plates [17]. It has been well established that upon illumination of incident light, oscillating electric current can be excited on a metallic surface. The surrounding electromagnetic field is then modulated by the irradiation of oscillating surface electric current. At resonant frequency, this effect is so significant that a thin layer of metallic structure can effectively tune the state of light. However, the underlying Lorentz resonance in metal is highly dispersive in nature, which limits its application to a specific narrow wave band. Overcoming the dispersion of metamaterials is essential for wide optical applications. On the other hand, it is known that the dielectric material interacts with light by accumulating an optical path within a certain thickness.

\footnotetext{
*To whom all correspondence should be addressed. muwang@nju.edu.cn

Published by the American Physical Society under the terms of the Creative Commons Attribution 3.0 License. Further distribution of this work must maintain attribution to the author $(s)$ and the published article's title, journal citation, and DOI.
}

This feature is effective over a broad bandwidth and has already been applied in antireflection coating and other optical devices [18-20]. By integrating a metallic metastructure and a dielectric interlayer, it is possible to realize a dispersion-free broadband device on the subwavelength scale, where the strong response of the metallic structures helps to decrease the device size while the dielectric interlayer helps to eliminate the dispersion simultaneously in both the amplitude and the phase difference of the reflected or transmitted light.

Thus far, much effort has been devoted to broaden the response frequency range of metallic metastructures [20-33]. For example, by superimposing different resonance modes within a unit cell, the bandwidth of the structure can be broadened [25-30]. Additionally, ultrathin, broadband, and highly efficient metamaterial-based terahertz polarization converters have been realized recently, which rotate a linear polarization state into its orthogonal one [20]. Furthermore, a broadband quarter-wave plate constructed with a periodic arrangement of metallic nanobricks above a homogeneous metallic film separated by an insulator layer has also been numerically demonstrated based on gap-plasmon resonators [33]. It is always interesting to identify the essential physics mechanism of these broadband designs. In this paper, we illustrate a general mechanism for manipulating the dispersion of a metastructure by integrating a metallic metamaterial, which possesses a strong yet dispersive interaction with light, with a dielectric interlayer, which has accumulative yet broadband interaction with light. In this way, the intrinsic dispersion of the metallic structures is perfectly cancelled out by the thickness-dependent dispersion of the dielectric spacing layer. As examples to apply this concept, a broadband quarter-wave plate and a half-wave plate are demonstrated. By proper selection of structural parameters, 
the polarization state of light can be freely tuned across a broad frequency range, and all of the polarization states on the Poincaré sphere can be realized dispersion free. In particular, the approach described here can be applied in designing other devices with dispersion-free features.

Our structure consists of a layer of metallic metastructure and a layer of perfect electric conductor (PEC) separated by a dielectric layer with thickness $d$. (Here, we take this layer as a vacuum in order to keep the formula simple. The details for the dielectric layer are provided in the Supplemental Material [34].) The response of each layer upon illumination of incident light $\vec{E}_{\text {inc }} e^{-i k z}$ is schematically shown in Fig. 1(a). For the metallic pattern without magnetic response [35], the irradiation can be expressed as $\vec{E}_{\mathrm{rad}} e^{-i k(z-d)}$ and $\vec{E}_{\mathrm{rad}} e^{i k(z-d)}$ for the waves propagating in $-z$ and $+z$ directions, respectively. The PEC layer acts as a perfect mirror. Therefore, the light reflected from the PEC plane can be regarded as the mirror image of the incident light together with the radiation of the mirror image of the metallic structure, as illustrated by the red arrows in Fig. 1(a). The mirror image of the incident light is expressed as $-\vec{E}_{\mathrm{inc}} e^{i k z}$. The mirror image of the metallic structure is located at $z=-d$ and its irradiation is expressed as two waves propagating in $-z$ and $+z$ directions, respectively, $-\vec{E}_{\mathrm{rad}} e^{-i k(z+d)}$ and $-\vec{E}_{\mathrm{rad}} e^{i k(z+d)}$. It follows that the total reflection is the superposition of all components of light propagating in the $+z$ direction,

$$
\vec{E}_{\mathrm{ref}}=-\vec{E}_{\mathrm{inc}}+\vec{E}_{\mathrm{rad}}\left(-e^{i k d}+e^{-i k d}\right) .
$$

Here we have ignored the common factor $e^{i k z}$ on both sides of Eq. (1). It should be pointed out that both $\vec{E}_{\text {rad }}$ and $\vec{E}_{\text {inc }}$ are the vectors and their polarizations are not necessarily identical. Therefore, the polarization of the reflected light can be different from that of the incident light. Because of the resonance nature of the metallic structure, $\vec{E}_{\text {rad }}$ is frequency dependent. The term $-e^{i k d}+e^{-i k d}$ reflects the conjugation relation between the radiation from the Lpatterned layer and its mirror image. As the wave vector $k$ is proportional to frequency, $-e^{i k d}+e^{-i k d}$ is frequency dependent as well. However, at the same time, the dispersion of $-e^{i k d}+e^{-i k d}$ depends on the separation $d$. By carefully selecting $d$, the dispersion of $-e^{i k d}+e^{-i k d}$ can compensate the dispersion of $\vec{E}_{\text {rad }}$. In this way, the dispersion-free feature is achieved [36].

To implement this idea, an array of gold L patterns, which is one of the simplest anisotropy resonators, is designed over a homogeneous, highly reflective silver mirror layer separated by a layer of $\mathrm{SiO}_{2}$, as shown in Fig. 1(b). Commercial software based on the finitedifference time-domain method is applied to simulate the response of the structure upon illumination of light (the details are provided in the Supplemental Material [34]). Before looking into the structure shown in Figs. 1(a) and 1(b), for comparison, it is interesting to investigate (a)

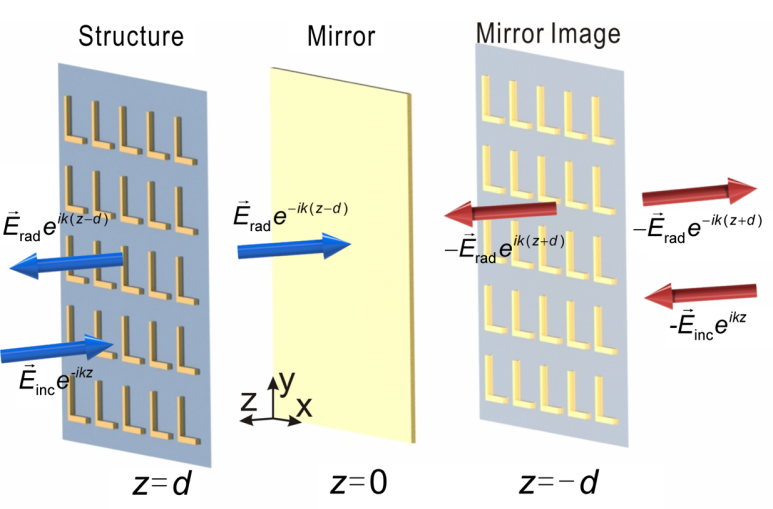

(b)

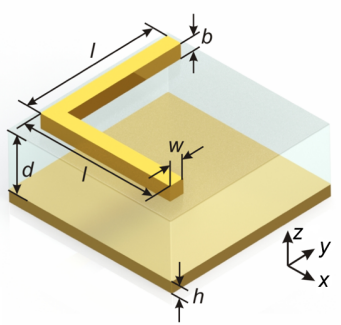

(c)
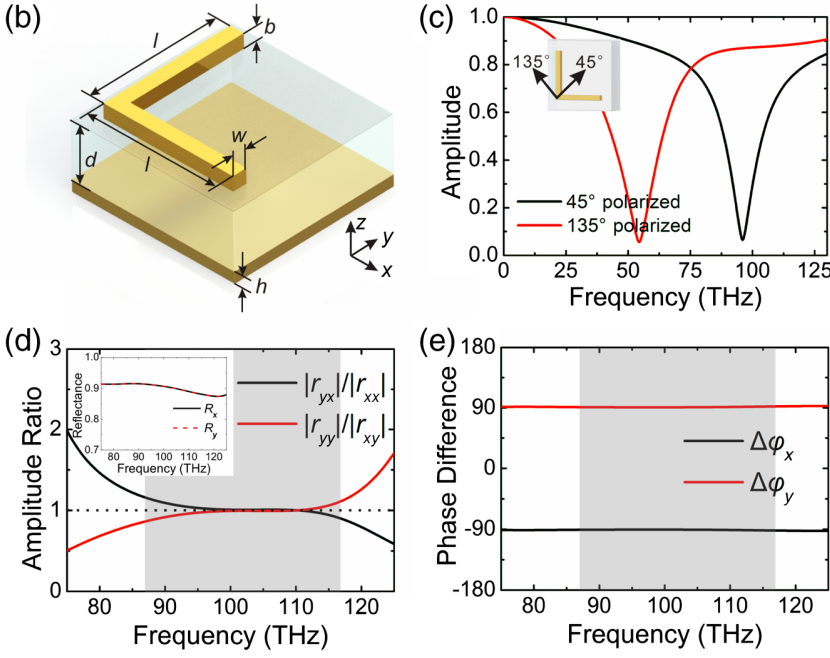

FIG. 1. (a) Schematics to show the interaction of light with each interface of the system made of an array of $L$ patterns $(z=d)$ in front of a perfect electric conductor $(z=0)$. The mirror image of the array is located at $z=-d$. (b) Structural parameters of the $\mathrm{L}$ pattern. The thickness of the silver mirror layer is $h=100 \mathrm{~nm}, \mathrm{SiO}_{2}$ layer thickness is $d=510 \mathrm{~nm}$. The length $l$, width $w$, and thickness $b$ of each arm of the L pattern are 1220, 120 , and $120 \mathrm{~nm}$, respectively. The lattice constant is $1550 \mathrm{~nm}$. (c) Simulated transmission of a layer of gold L-pattern array only, without the silver mirror layer underneath. The black and red lines are the normalized amplitude of the electric component of the transmitted light of $45^{\circ}$-and $135^{\circ}$-polarized incidence. (d) Simulated amplitude ratio of the reflected light, which becomes unity in the shaded frequency band. The inset shows the total reflectance. (e) Simulated phase difference of the reflected light, which is either $90^{\circ}$ or $-90^{\circ}$.

the transmittance of an independent array of $\mathrm{L}$ patterns without the mirror layer. When the incident light is polarized along $45^{\circ}$ or $135^{\circ}$, due to the symmetry of the structure, the polarization of the transmitted and the reflected light does not change. The normalized electric components of the transmitted light are shown in Fig. 1(c). The symmetric resonance mode and the antisymmetric resonance mode are excited by the $45^{\circ}$ and $135^{\circ}$ polarized light at the higher and lower frequencies, respectively, which has been reported [11]. Apparently, the bare $L$-patterned layer acts as a highly dispersive anisotropy 
resonator. When a homogeneous silver mirror layer is introduced beneath the structure at $z=0$, the transmission of the structure is zero. The simulated total reflectance $R_{x(y)}$ is shown in the inset of Fig. 1(d), where $R_{x(y)}$ is about $90 \%$, indicating that the structure is an excellent reflector. The subscript in $R_{x(y)}$ represents the polarization of incident light. The amplitude ratio between the two components of the reflected light with both $x$ - and $y$-polarized incidence are calculated [Fig. 1(d)]. $r_{i j}$ stands for the complex amplitude of the $i$ component of the reflected light induced by $j$-polarized incidence $(i, j=x, y)$. The phase difference $\Delta \varphi_{i(j)}$ is defined as the difference between the phase of the $y$ component and that of the $x$ component of the reflected light, with the subscript of $\Delta \varphi_{i(j)}$ standing for the polarization of incident light. For the $x$-polarized incidence, in a broad frequency band ranging from 87 to $117 \mathrm{THz}$, the amplitude ratio remains unity and the phase difference $\Delta \varphi_{x}$ is $-90^{\circ}$, as illustrated in Figs. 1(d) and 1(e). This means that the reflected light has been changed to a right-handed circularly polarized light. For the $y$-polarized incidence, a left-handed circularly polarized reflected light is generated in the same frequency range.

The gold $L$ patterns fabricated by electron beam lithography (EBL) are shown in Fig. 2(a), beneath which a 510-nm-thick $\mathrm{SiO}_{2}$ separation layer and a 100 -nm-thick homogeneous silver mirror layer have been fabricated by magnetron sputtering on a silicon substrate. The measured reflectance of the sample is larger than 0.85 in the response frequency [Fig. 2(b)]. From 86 to $116 \mathrm{THz}$, the amplitude ratio remains unity for both the $x$ - and $y$-polarized incidence [Fig. 2(c)]. The phase difference is $-90^{\circ}$ for

(a)
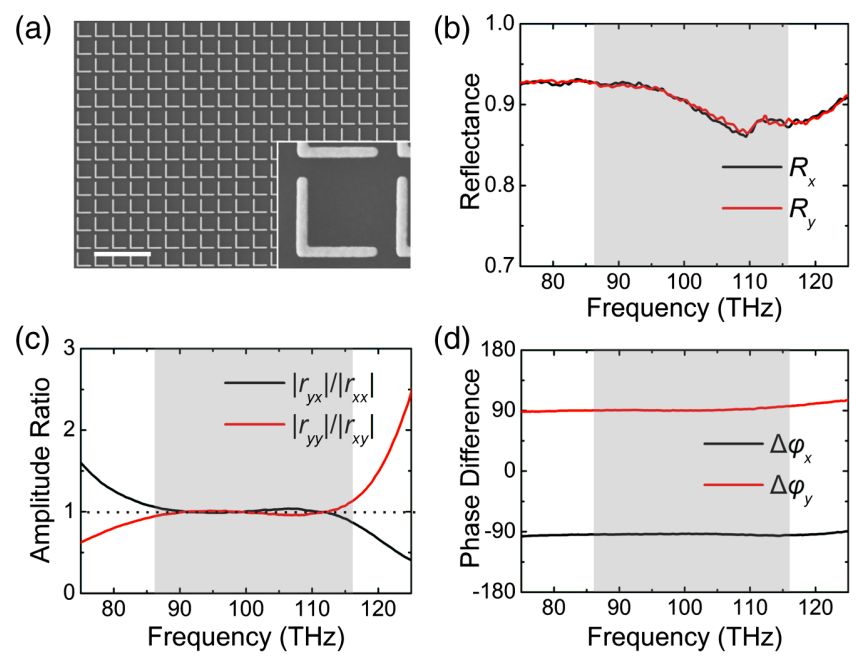

FIG. 2. (a) Field-emission scanning electron micrograph of the array of gold L patterns fabricated by electron beam lithography. The bar represents $5 \mu \mathrm{m}$. (b) Experimentally measured reflectance for the $x$ - and $y$-polarized incident light. (c),(d) Experimentally measured amplitude ratio and phase difference of the sample under the illumination of $x$ - and $y$-polarized light, respectively. the $x$-polarized incidence, and it becomes $90^{\circ}$ for the $y$-polarized incidence. Therefore, a dispersion-free quarter-wave plate is experimentally realized and the bandwidth reaches about $30 \%$ of the central frequency.

The electromagnetic response of our structure can be theoretically analyzed. For simplicity, here we ignore the loss of metal and take the refractive index of $\mathrm{SiO}_{2}$ as 1.0 in the calculation. The irradiation field from the induced surface electric current is proportional to the total external electric field that excites the resonance of the surface electric current, which is the superposition of the electric components of the incident light and the light reflected by the mirror plane. The electric field of light is expressed as a column vector, with subscripts $x$ and $y$ representing the $x$ and $y$ components of the electric field, respectively. It follows that

$$
\left(\begin{array}{c}
E_{\mathrm{rad}, x} \\
E_{\mathrm{rad}, y}
\end{array}\right)=\left(\begin{array}{cc}
\sigma_{1} & \sigma_{2} \\
\sigma_{2} & \sigma_{1}
\end{array}\right)\left(\begin{array}{c}
E_{\mathrm{ext}, x} \\
E_{\mathrm{ext}, y}
\end{array}\right),
$$

where $\vec{E}_{\text {ext }}$ is the total external field on the plane of $L$ patterns. The symmetry of the $\sigma$ matrix is due to the mirror symmetry of the structure in the diagonal direction. By taking the L-patterned unit as two connected RLC circuits [37], the elements in the $\sigma$ matrix can be described by the symmetric and antisymmetric modes as

$$
\begin{aligned}
\sigma_{1} & =\frac{i \gamma \omega}{2\left(\omega_{0}^{2}-\omega^{\prime 2}-\omega^{2}-i \gamma \omega\right)}+\frac{i \gamma \omega}{2\left(\omega_{0}^{2}+\omega^{\prime 2}-\omega^{2}-i \gamma \omega\right)}, \\
\sigma_{2} & =-\frac{i \gamma \omega}{2\left(\omega_{0}^{2}-\omega^{\prime 2}-\omega^{2}-i \gamma \omega\right)}+\frac{\left.i \omega_{0}^{2}+\omega^{\prime 2}-\omega^{2}-i \gamma \omega\right)}{2(3)}
\end{aligned}
$$

where $\omega_{0}$ denotes the response frequency of each individual arm, $\omega^{\prime}$ denotes the interaction between them, and $\gamma$ represents the effective resistance.

For a bare layer of $L$ patterns only, the excitation field for the resonance of the $L$ pattern $\vec{E}_{\text {ext }}$ is merely the incident light $\vec{E}_{\text {inc }}$. Meanwhile, the transmission is the superposition of the incident light and the radiation light based on Eq. (2). For the integrated structure, however, the radiation of the array of $L$ patterns is excited by both the incident light and the reflected light from the PEC plane, as illustrated in Fig. 1(a). It follows that

$$
\vec{E}_{\mathrm{ext}}=\vec{E}_{\mathrm{inc}} e^{-i k d}-\vec{E}_{\mathrm{inc}} e^{i k d}-\vec{E}_{\mathrm{rad}} e^{2 i k d} .
$$

From Eqs. (1)-(4), we can get the reflected field $\vec{E}_{\text {ref }}$. Suppose the incident light $\left(\begin{array}{l}E_{\operatorname{inc} x} \\ E_{\operatorname{inc} y}\end{array}\right)$ is $x$-polarized and is denoted as $\left(\begin{array}{l}1 \\ 0\end{array}\right)$. The parameters in Eq. (3) $\left(\omega_{0}, \omega^{\prime}\right.$, and $\left.\gamma\right)$ can be retrieved from the simulation of a single-layered $\mathrm{L}$ 
pattern (see Supplemental Material for details [34]). The relationship among the amplitude ratio of the two components of the reflected light, the frequency, and the separation layer thickness $d$ is illustrated in Fig. 3(a). The color indexes the amplitude ratio of the reflected light. One may find that when the thickness of the separation layer $d$ is selected as $911 \mathrm{~nm}$, the color does not vary with frequency, and the dispersion-free feature is realized, as marked by the dashed line. The calculated amplitude ratio of the reflected light and the phase difference between the $y$ and $x$ components at $d=911 \mathrm{~nm}$ are shown in Fig. 3(b), which is consistent with the experimental results shown in Figs. 2(c) and 2(d). Therefore, we conclude that with proper selection of interlayer separation $d$, the amplitude ratio can become frequency independent. One may notice that here the interlayer thickness $(d=911 \mathrm{~nm})$ is larger than the design in Fig. 1(b). The reason is that here we take the refractive index of the dielectric layer as 1.0 for simplicity of calculation, yet $\mathrm{SiO}_{2}$ possesses a larger refractive index. As elucidated in the Supplemental Material [34], once the refractive index of $\mathrm{SiO}_{2}$ in the observation frequency regime is taken as $n=1.41$, the resulting separation becomes $d=582 \mathrm{~nm}$, which is in reasonable agreement with our design in Fig. 1(b).

To understand the mechanism better, it is interesting to identify how the radiation of the structure $\vec{E}_{\text {rad }}$ and the phase-conjugation term $-e^{i k d}+e^{-i k d}$ in Eq. (1) change with frequency, respectively. In our calculation, the radiation of the metallic structure is derived directly from Eq. (2). The dependence of the amplitude of the $x$ and $y$ components of $\vec{E}_{\mathrm{rad}}$ and $\left|-e^{i k d}+e^{-i k d}\right|$ on frequency is
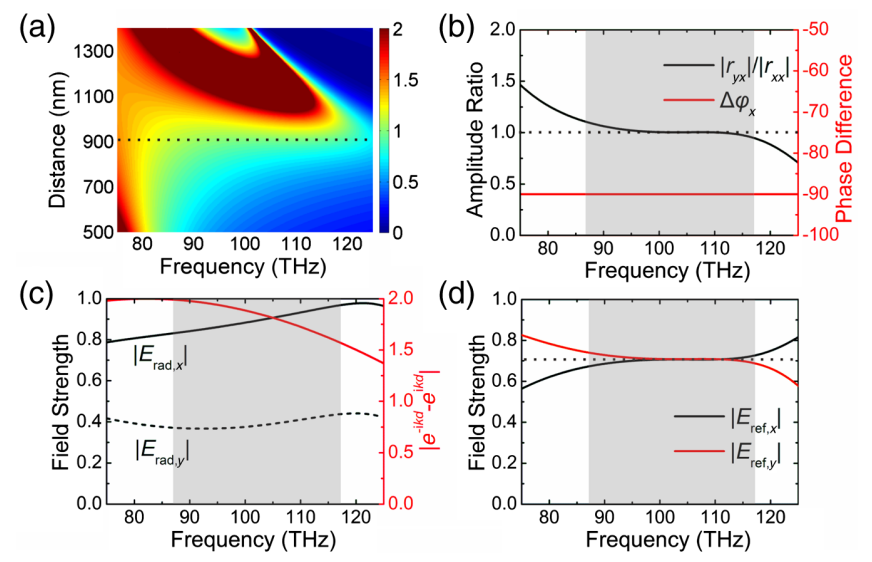

FIG. 3. (a) Relationship of the interlayer thickness $d$, the frequency and the amplitude ratio of the reflected light. The color represents the amplitude ratio of the reflected light. (b) Calculated amplitude ratio and phase difference of the reflected light under the $x$-polarized incident light, which is similar to that shown in Figs. 1(d) and 1(e). (c) Amplitude of the $x$ and $y$ components of the irradiation of the structure and the amplitude of $\left|-e^{i k d}+e^{-i k d}\right|$, as a function of frequency. (d) Calculated amplitude of the $x$ and $y$ components of the reflected light. shown in Fig. 3(c). One may find that in the frequency band we are interested in, the $x$ and $y$ components of the amplitude of the irradiation $\vec{E}_{\text {rad }}$ keep increasing, whereas $\left|-e^{i k d}+e^{-i k d}\right|$ decreases when frequency is increased. According to Eq. (1), normalized $x$ and $y$ components of the reflected light can be expressed as $E_{\mathrm{rad}, x}\left(-e^{i k d}+\right.$ $\left.e^{-i k d}\right)-1$ and $E_{\mathrm{rad}, y}\left(-e^{i k d}+e^{-i k d}\right)$, respectively. By selecting suitable separation $d$, the increase of $\left|E_{\text {rad, } y}\right|$ can be compensated by the decrease of $\left|-e^{i k d}+e^{-i k d}\right|$ within a certain frequency range. Hence, the amplitude of the $y$ component of the reflected light becomes dispersion free. Similarly, the amplitude of the $x$ component of reflected light is frequency independent as well [Fig. 3(d)].

The dispersion-free feature of the phase difference, however, originates from the geometrical symmetry of the structure. When the polarization of incident light is along $45^{\circ}$, due to structural symmetry, the polarization of the reflected light resumes the original state and the amplitude ratio of the reflected light is unity when the electromagnetic loss is ignored. The same situation occurs for $135^{\circ}$-polarized incidence. For $x$-polarized incidence, the amplitudes of $45^{\circ}$ - and $135^{\circ}$-polarized components are identical. It follows that the amplitudes of reflected light in these two directions are identical as well. In the $x-y$ coordination system, the components of the reflected light in $45^{\circ}$ and $135^{\circ}$ orientations are expressed as $\frac{\sqrt{2}}{2}\left(r_{x x}+r_{y x}\right)$ and $\frac{\sqrt{2}}{2}\left(-r_{x x}+r_{y x}\right)$, respectively. Since $r_{x x}$ and $r_{y x}$ represent the complex amplitude of two components of the reflected light, the fact that $\left|r_{x x}+r_{y x}\right|$ equals $\left|-r_{x x}+r_{y x}\right|$ indicates that $r_{x x}$ and $r_{y x}$ are perpendicular to each other in the complex plane. Therefore, the absolute value of the phase difference for $r_{x x}$ and $r_{y x}$ is $90^{\circ}$. Similarly, the value of the phase difference of $r_{x y}$ and $r_{y y}$ is $90^{\circ}$.

With this phase-conjugation scheme, a dispersion-free device with other functionalities can be achieved by tuning the structural parameters. For example, by changing the length and width of each arm of the $L$-patterned structure to 800 and $150 \mathrm{~nm}$, respectively, and at the same time shrinking the lattice constant to $1400 \mathrm{~nm}$ and the thickness of the $\mathrm{SiO}_{2}$ layer to $420 \mathrm{~nm}$, an ultrabroadband half-wave plate can be realized. Figure 4(a) shows the simulated results, where $x$-polarized ( $y$-polarized) incident light is turned to the $y$-polarized ( $x$-polarized) reflected light, and the intensity of the $x(y)$ component in reflection is almost zero in the frequency band from 70 to $145 \mathrm{THz}$. The structure has been experimentally fabricated, and the two components of the reflection under illumination of the $x$ - and $y$-polarized incidence are illustrated in Fig. 4(b). Meanwhile, the bandwidth of the half-wave plate reaches about $67 \%$ of the central wavelength. The light intensity ratio is shown in Fig. 4(c). Within the response frequency range, the ratio is around $10^{2}-10^{3}$, indicating that most of the energy of the $x$-polarized ( $y$-polarized) incidence has been transferred to the $y(x)$ component of the reflected light. 


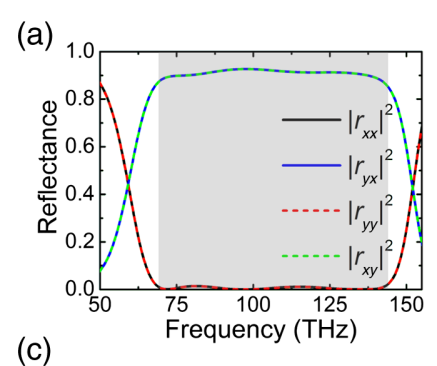

(c)
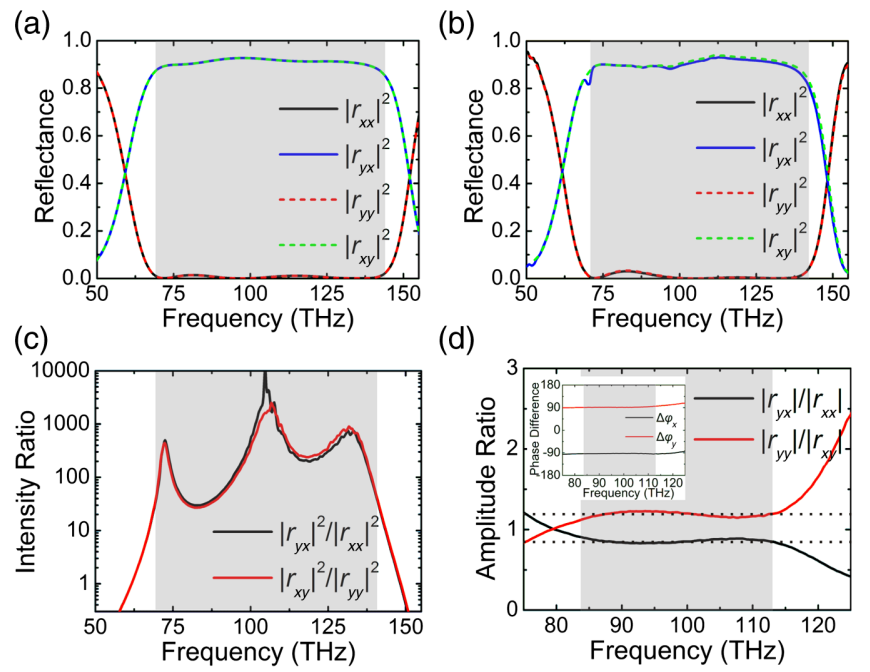

(d)

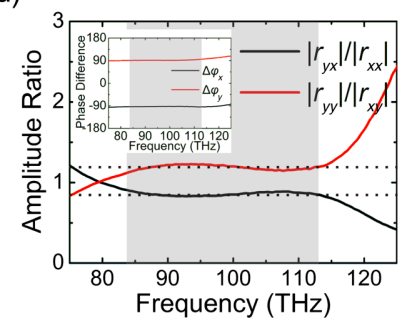

tuning the polarization state of light only. Instead, we expect that it can be applied in designing other devices with broadband features and can help to make the ultimate dream of mastering light on the nanoscale come true.

The authors acknowledge support from the MOST of China (Grants No. 2010CB630705 and No. 2012CB921502), the NSF of China (Grants No. 50972057, No. 11034005, No. 11204127, and No. 61077023), the MOE of China (SRFDP No. 20120091120033), and partial support by Jiangsu Province (Grant No. BK2012301).

[1] C. M. Soukoulis and M. Wegener, Past Achievements and Future Challenges in the Development of ThreeDimensional Photonic Metamaterials, Nat. Photonics 5, 523 (2011).

FIG. 4. (a) Simulated reflectance for a broadband half-wave plate. (b) Experimentally measured reflectance of the fabricated sample, which is in good agreement with simulations. (c) Measured intensity ratio between the $x$ and $y$ components of reflectance. (d) The wave plate to generate the elliptical polarized light has been fabricated. The measurements show that the amplitude ratio between the two components of the reflected light deviates from 1 in the shaded region, whereas the phase difference (inset) between the two orthogonal components remains $90^{\circ}$.

The dispersion-free quarter- and half-wave plates allow turning $50 \%$ and $100 \%$ of the reflected power from one polarization to the perpendicular polarization of the reflected light over a broad frequency band. Moreover, the amplitude ratio between the two perpendicular components of the reflected light can be continuously tuned, and a dispersion-free wave plate with nonunity amplitude ratio can be realized by selecting the structural parameters of the $L$-patterned array. For example, when the length and width of each arm of the $L$ pattern are selected as 1290 and $155 \mathrm{~nm}$, respectively, and we keep the other parameters identical to those in Fig. 1(b), the amplitude ratio becomes 0.84 and 1.19 for $x$ - and $y$-polarized incidence, respectively, in the range of $84-113 \mathrm{THz}$, as illustrated in Fig. 4(d). Meanwhile, the phase difference remains $-90^{\circ}$ and $90^{\circ}$ [see the inset of Fig. 4(d)]. In this way, a dispersionfree wave plate for elliptical polarized light is achieved.

Controlling the state of light has been an important topic in nanophotonics. Metamaterials and plasmonics provide the opportunity to miniaturize the devices and realize different wave plate functionalities with new principles. By integrating metallic metastructures and dielectric interlayer, the intrinsic dispersion generated by the resonance of metallic structures is compensated by the interlayerthickness-sensitive dispersion of the dielectric interlayer, so the dispersion-free optical functionalities can be realized in a very restricted space. We also point out that the principle presented here is universal in physics, and is not limited to

[2] Y. Liu and X. Zhang, Metamaterials: A New Frontier of Science and Technology, Chem. Soc. Rev. 40, 2494 (2011).

[3] D. R. Smith, W. J. Padilla, D. C. Vier, S. C. Nemat-Nasser, and S. Schultz, Composite Medium with Simultaneously Negative Permeability and Permittivity, Phys. Rev. Lett. 84, 4184 (2000).

[4] R. A. Shelby, D. R. Smith, and S. Schultz, Experimental Verification of a Negative Index of Refraction, Science 292, 77 (2001).

[5] J. Valentine, S. Zhang, T. Zentgraf, E. Ulin-Avila, D. A. Genov, G. Bartal, and X. Zhang, Three-Dimensional Optical Metamaterial with a Negative Refractive Index, Nature (London) 455, 376 (2008).

[6] N. Fang, H. Lee, C. Sun, and X. Zhang, Sub-DiffractionLimited Optical Imaging with a Silver Superlens, Science 308, 534 (2005).

[7] Z. Liu, H. Lee, Y. Xiong, C. Sun, and X. Zhang, Far-Field Optical Hyperlens Magnifying Sub-Diffraction-Limited Objects, Science 315, 1686 (2007).

[8] I. I. Smolyaninov, Y.-J. Hung, and C. C. Davis, Magnifying Superlens in the Visible Frequency Range, Science 315, 1699 (2007).

[9] R. G. Hunsperger, Integrated Optics:Theory and Technology (Springer, New York, 2009), 6th ed.

[10] A. V. Kildishev, A. Boltasseva, and V. M. Shalaev, Planar Photonics with Metasurfaces Science 339, 1232009 (2013).

[11] N. Yu, P. Genevet, M. A. Kats, F. Aieta, J.-P. Tetienne, F. Capasso, and Z. Gaburro, Light Propagation with Phase Discontinuities: Generalized Laws of Reflection and Refraction, Science 334, 333 (2011).

[12] X. Ni, N. K. Emani, A. V. Kildishev, A. Boltasseva, and V. M. Shalaev, Broadband Light Bending with Plasmonic Nanoantennas, Science 335, 427 (2012).

[13] A. Pors, O. Albrektsen, I. P. Radko, and S. I. Bozhevolnyi, Gap-Plasmon-Based Metasurfaces for Total Control of Reflected Light, Sci. Rep. 3, 2155 (2013).

[14] S. Sun, K.-Y. Yang, C.-M. Wang, T.-K. Juan, W. T. Chen, C. Y. Liao, Q. He, S. Xiao, W.-T. Kung, G.-Y. Guo, L. Zhou, and D.P. Tsai, High-Efficiency Broadband Anomalous Reflection by Gradient Meta-Surfaces, Nano Lett. 12, 6223 (2012). 
[15] A. Pors, M. G. Nielsen, R. L. Eriksen, and S. I. Bozhevolnyi, Broadband Focusing Flat Mirrors Based on Plasmonic Gradient Metasurfaces, Nano Lett. 13, 829 (2013).

[16] P. Genevet, N. Yu, F. Aieta, J. Lin, M. A. Kats, R. Blanchard, M. O. Scully, Z. Gaburro, and F. Capasso, Ultrathin Plasmonic Optical Vortex Plate Based on Phase Discontinuities, Appl. Phys. Lett. 100, 013101 (2012).

[17] N. Yu, F. Aieta, P. Genevet, M. A. Kats, Z. Gaburro, and F. Capasso, A Broadband, Background-Free Quarter-Wave Plate Based on Plasmonic Metasurfaces, Nano Lett. 12, 6328 (2012).

[18] M. Born and E. Wolf, Principles of Optics (Cambridge University Press, Cambridge, England,, 1999), 7th ed.

[19] H.-T. Chen, J. Zhou, J. F. O'Hara, F. Chen, A. K. Azad, and A. J. Taylor, Antireflection Coating Using Metamaterials and Identification of Its Mechanism, Phys. Rev. Lett. 105, 073901 (2010).

[20] N. K. Grady, J. E. Heyes, D. R. Chowdhury, Y. Zeng, M. T. Reiten, A. K. Azad, A. J. Taylor, D. A. R. Dalvit, and H.-T. Chen, Terahertz Metamaterials for Linear Polarization Conversion and Anomalous Refraction, Science 340, 1304 (2013).

[21] J. K. Gansel, M. Thiel, M. S. Rill, M. Decker, K. Bade, V. Saile, G. von Freymann, S. Linden, and M. Wegener, Gold Helix Photonic Metamaterial as Broadband Circular Polarizer, Science 325, 1513 (2009).

[22] J. K. Gansel, M. Latzel, A. Frolich, J. Kaschke, M. Thiel, and M. Wegener, Tapered Gold-Helix Metamaterials as Improved Circular Polarizers, Appl. Phys. Lett. 100, 101109 (2012).

[23] C. Wu, H. Li, X. Yu, F. Li, H. Chen, and C. T. Chan, Metallic Helix Array as a Broadband Wave Plate, Phys. Rev. Lett. 107, 177401 (2011).

[24] Z. Y. Yang, M. Zhao, P. X. Lu, and Y. F. Lu, Ultrabroadband Optical Circular Polarizers Consisting of DoubleHelical Nanowire Structures, Opt. Lett. 35, 2588 (2010).

[25] Y. Zhao, M. A. Belkin, and A. Alù, Twisted Optical Metamaterials for Planarized Ultrathin Broadband Circular Polarizers, Nat. Commun. 3, 870 (2012).

[26] F. Ding, Y. Cui, X. Ge, Y. Jin, and S. He, Ultra-Broadband Microwave Metamaterial Absorber, Appl. Phys. Lett. 100, 103506 (2012).

[27] D. R. Chowdhury, R. Singh, M. Reiten, H.-T. Chen, A. J. Taylor, J. F. O'Hara, and A. K. Azad, A Broadband Planar
Terahertz Metamaterial with Nested Structure, Opt. Express 19, 15817 (2011).

[28] N. R. Han, Z. C. Chen, C. S. Lim, B. Ng, and M. H. Hong, Broadband Multilayer Terahertz Metamaterials Fabrication and Characterization on Flexible Substrates, Opt. Express 19, 6990 (2011).

[29] Z. Wei, Y. Cao, Y. Fan, X. Yu, and H. Li, Broadband Polarization Transformation via Enhanced Asymmetric Transmission through Arrays of Twisted Complementary Split-Ring Resonators, Appl. Phys. Lett. 99, 221907 (2011).

[30] X. Xiong, W.-H. Sun, Y.-J. Bao, R.-W. Peng, M. Wang, C. Sun, X. Lu, J. Shao, Z.-F. Li, and N.-B. Ming, Switching the Electric and Magnetic Responses in a Metamaterial, Phys. Rev. B 80, 201105(R) (2009).

[31] Y. Zhao and A. Alù, Tailoring the Dispersion of Plasmonic Nanorods To Realize Broadband Optical Meta-Wave Plates, Nano Lett. 13, 1086 (2013).

[32] P. E. Sieber and D. H. Werner, Reconfigurable Broadband Infrared Circularly Polarizing Reflectors Based on Phase Changing Birefringent Metasurfaces, Opt. Express 21, 1087 (2013).

[33] A. Pors and S. I. Bozhevolnyi, Efficient and Broadband Quarter-Wave Plates by Gap-Plasmon Resonators, Opt. Express 21, 2942 (2013).

[34] See Supplemental Material at http://link.aps.org/ supplemental/10.1103/PhysRevX.4.021026 for the details of the physical modeling describing our system and the simulation results.

[35] M. Albooyeh, D. Morits, and S. A. Tretyakov, Effective Electric and Magnetic Properties of Metasurfaces in Transition from Crystalline to Amorphous State, Phys. Rev. B 85, 205110 (2012).

[36] For the scenario that the interlayer is another dielectric material instead of air, the expression for the reflected light becomes more complicated than Eq. (1) due to reflection and refraction at the dielectric-air boundary, yet the fundamental principle to achieve dispersion-free feature is the same.

[37] S.-C. Jiang, X. Xiong, P. Sarriugarte, S.-W. Jiang, X.-B. Yin, Y. Wang, R.-W. Peng, D. Wu, R. Hillenbrand, X. Zhang, and M. Wang, Tuning the Polarization State of Light via Time Retardation with a Microstructured Surface, Phys. Rev. B 88, 161104(R) (2013). 